\title{
Independence of neophobia and taste aversion learning
}

\author{
NORMAN S. BRAVEMAN and PAUL S. JARVIS \\ Memorial University of Newfoundland, St. John's, Newfoundland, Canada
}

\begin{abstract}
Animals were presented with (1) one solution which differed from that of the test solution, (2) a series of distinctly flavored solutions whose flavors differed from that of the test solution, or (3) with a flavored solution whose flavor was the same as that of the test solution. When animals received the solution whose flavor was the same as that of the test solution prior to a test for neophobia and prior to a conditioning trial, neophobia was reduced and aversions were weakened. However, when animals received a solution or a series of solutions whose flavors differed from that of the test solution, neophobia was reduced but conditioned aversions were unaffected. Presentations of solutions that differed from the test solution following aversion formation left the association between the taste of the test solution and the effects of the aversion-inducing treatment intact. In a final experiment it was discovered that neophobia was reduced as much when animals drank solutions whose flavors changed every third day as when they drank the same solution throughout testing.
\end{abstract}

It has been observed (e.g., Revusky, 1971; Siegel, 1974) that familiarizing animals with the taste of a solution can have two effects. On the one hand, animals that are familiar with the taste of a solution at the time of testing tend to consume more of it (i.e., they are less neophobic) than animals for whom the taste is novel (e.g., Barnett, 1963). A second effect of taste familiarity which has also been well documented (e.g., Revusky, 1971), is that conditioned taste aversions are weakened when animals are familiar with the taste of the test solution at the time of training.

Recently, however, it has been reported that neophobia can also be reduced by familiarizing animals with a solution whose taste is different from that of the test solution. In one experiment, for example, it was discovered that rats preexposed to one solution (coffee or vinegar) drank more of a second solution (vinegar or coffee, respectively) than animals not preexposed to a novel tasting solution (Siegel, 1974). Similar results were reported by Capretta, Petersik, and Stewart (1975) in an experiment in which immature animals that received a series of distinctively flavored novel solutions were less neophobic to another novel solution than

The authors express their thanks to Joan Crane for her able assistance with Experiment 2. This research was supported, in part, by a grant from the Dean of Science (MUN) and by Grant A-8334 from the National Research Council of Canada to the first author. Portions of this paper were presented at the 17th Annual Meeting of the Psychonomic Society, St. Louis, Missouri 1976. Requests for reprints should be addressed to N.S. Braveman, Department of Psychology, Memorial University of Newfoundland, St. John's, Newfoundland, Canada. animals that had not been preexposed to the variable series of solutions.

Since both neophobia and conditioning are reduced if animals are familiar with the taste of the test solution and since familiarity with solutions other than the test solution reduces neophobia, it is possible that familiarity with solutions other than the test solution might also result in weakened conditioned taste aversions. Unfortunately, neither Siegel nor Capretta et al. reported whether or not this was indeed the case. Thus, the aim of the first experiment was to discover whether or not animals that were familiarized with one distinctively flavored solution or a set of solutions exhibited reduced neophobia and formed weaker taste aversions to another, distinctively different, flavored solution.

\section{EXPERIMENT 1}

\section{Method}

Subjects. The subjects in this experiment were 112 male, experimentally naive hooded rats derived from the Long-Evans strain and obtained from a commercial breeder. The animals were housed in standard stainless steel laboratory cages whose dimensions were $19 \times 19 \times 37 \mathrm{~cm}$. The range of weights for the animals at the start of the experiment was between 250 and $300 \mathrm{~g}$.

Apparatus. All phases of the experiment were conducted in the animals' home cages. The solutions were $.9 \%(w / v)$ sodium chloride $(\mathrm{Na}), .15 \%(\mathrm{w} / \mathrm{v})$ sodium saccharin $(\mathrm{S}), .75 \%(\mathrm{w} / \mathrm{v})$ coffee (C), 3\% (v/v) lemon extract (L), 3\% (v/v) almond extract (A), and tap water (W). These solutions were presented to the animals in measured amounts via standard $500-\mathrm{ml}$ water bottles which had been fitted with metal spouts.

Procedure. Following adaptation to a feeding/drinking schedule, on which animals received ad-lib access to food and $10 \mathrm{ml}$ of tap water per day from a graduated drinking bottle for 2 weeks, 
the animals were randomly assigned to one of seven groups of 16 subjects each. Animals in Group $W$ received eight additional daily presentations of $10 \mathrm{ml}$ of tap water. Those in Group S, Group C, Group A, Group L, and Group Na received $10 \mathrm{ml}$ of saccharin-, coffee-, almond-, lemon-, or $\mathrm{NaCl}$-flavored water, respectively, on each of the eight presentatons. Each animal in Group $\mathrm{V}$ received daily $10-\mathrm{ml}$ presentations of saccharin-, almond-, lemon-, and coffee-flavored water on two separate occasions. The sequence of daily presentations was random for each of the animals in this group.

On Day 23 of the experiment, all animals in each group received the $\mathrm{NaCl}$ solution. Eight of the animals in each group were given a neophobia test by allowing them 10 min to drink the $\mathrm{NaCl}$ solution. These animals were used to assess the effects of the various preexposure experiences on neophobia by measuring the amount of the $\mathrm{NaCl}$ solution that they consumed to the nearest $1.0 \mathrm{ml}$. The remaining animals were given an aversion training trial by injecting them, intraperitoneally, with $1 \%$ body weight of $.3 \mathrm{M}$ (12.72 g/liter) of $\mathrm{LiCl} 60-90 \mathrm{sec}$ after they had finished drinking $10 \mathrm{ml}$ of the $\mathrm{NaCl}$ solution. For the next 2 days the conditioned animals received $10 \mathrm{ml}$ of tap water in their home cages and then, on Day 26, they were given an aversion test by allowing them 10 min access to the $\mathrm{NaCl}$ solution. The amount consumed was measured to the nearest $1.0 \mathrm{ml}$.

\section{Results}

The results of Experiment 1 are summarized in Figure 1. Panel A shows the amount consumed by animals on the test for neophobia. A one-way ANOVA on the amount consumed revealed an overall significant difference among the groups $[F(6,49)$ $=7.46, \mathrm{p}<.05]$. Newman-Keuls comparisons on the means revealed that all groups drank reliably more than the water-preexposed animals (all ps $<.05$ ) and that none of these groups differed from each other. Thus, preexposure to the test solution or to any one or combination of the other flavored solution reduced neophobia by an equivalent amount.

Results of the Day 26 aversion tests are summarized in Panel B of Figure 1 and were also
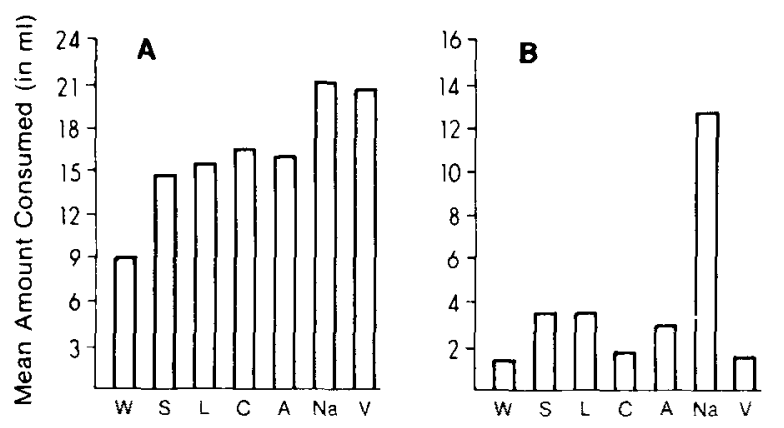

PREEXPOSURE CONDITIONS

Figure 1. Amount of salt water consumed by animals that had been preexposed to tap water (W), saccharin (S), lemon (L), coffee (C), almond $(A)$, sodium chloride $(\mathrm{Na})$, or solutions whose tastes varied (V) on each of 8 days. Panel $A$ depicts the results of a test for neophobia and Panel $B$ depicts the results of a test for conditioned taste aversion. analyzed by a one-way ANOVA which revealed that there were reliable differences among the groups $[F(6,49)=28.78, p<.01]$. Newman-Keuls comparisons among these means revealed that all groups drank reliably less than Group $\mathrm{Na}$, the animals that had been preexposed to the $\mathrm{NaCl}$ solution (all ps $<.01$ ), yet none of the remaining groups differed from each other. This outcome shows that all preexposed groups, except those that were familiar with the $\mathrm{NaCl}$ solution, formed aversions to the $\mathrm{NaCl}$ solution that were as strong as those that were formed by animals that had been preexposed to the highly familiar tap water. Taken together, the results of this experiment extend those reported by Capretta et al. and by Siegel in that they show that neophobia can be reduced by preexposure to any one of several flavored solutions, including a variable series of different flavors, but that aversions are reduced only when animals are preexposed to the taste of the solution that is used during training. ${ }^{1}$

\section{EXPERIMENT 2}

The results of the previous experiment showed that familiarization to the taste of a solution can reduce neophobia to another solution without interfering with associations between the taste of that solution and the aversive aftereffects of a lithium chloride injection. This finding suggests that animals may learn that a particular solution is a safe one even though they have not previously tasted it and that such learning does not appear to disrupt associations between the taste of that solution and the aversive aftereffects of a lithium chloride aversion.

In the present experiment we examined another aspect of this question by investigating whether experience with solutions other than the test solution influences already established associations between the taste of a solution and the aversive aftereffects of a lithium chloride injection. This was accomplished by first establishing a conditioned taste aversion to the $\mathrm{NaCl}$ test solution and then administering extinction trials using the various solutions employed in the previous experiment. If, as suggested by the results of the first experiment, exposure to tastes other than that which characterize the test solution prior to conditioning does not interfere with tastelithium chloride associations, then exposure to these other tastes during extinction should also leave an already established association intact. Thus, following extinction trails with one of the novel flavored solutions or with a random combination of these solutions, animals should exhibit aversions that are no weaker than those observed in animals that receive extinction trials with neutral tap water. At the same time, however, evidence of extinction of 
the conditioned aversion should be seen in animals that receive extinction trials with the same $\mathrm{NaCl}$ solution that is used during conditioning.

\section{Method}

The present experiment was the same as Experiment 1 in all unspecified details.

Subjects. The subjects in the present experiment were 38 male, experimentally naive hooded rats from the same strain and supplier as in the previous experiment.

Procedure. Following 14 days of adaptation to a $10-\mathrm{ml}$ per day drinking schedule, all animals were presented with a noveltasting salt solution on Day 15 and then injected, immediately, with a $.3-\mathrm{M}$ solution of $\mathrm{LiCl}$ which was equal in volume to $1 \%$ of body weight. On the next day, the animals were randomly assigned to one of seven groups. These groups differed from each other in terms of the type of solution that the animals received during their regular drinking sessions on Days 16-23. Animals in Group WAT $(\mathbf{N}=5)$ received tap water, those in Group SAC $(N=5)$ received saccharin-flavored water, those in Group LEM $(N=5)$ received lemon-flavored water, those in Group $\operatorname{COF}(N=7)$ recieved coffee-flavored water, those in Group $\operatorname{ALM}(N=6)$ received almond-flavored water, those in Group SAL $(\mathrm{N}=5)$ received $\mathrm{NaCl}$ water, and those in Group VAR $(N=5)$ received a variable series of two presentations each of saccharin-, almond-, coffee-, or lemon-flavored water. The additional animals in Groups COF and ALM resulted from the random assignment of extra animals to these two groups. Consumption on Days $16-23$ was limited to $10 \mathrm{ml}$ of the relevant solution. On Day 24 , all animals were presented, once again, with the $\mathrm{NaCl}$ water for $10 \mathrm{~min}$, and the amount consumed was measured to the nearest $1.0 \mathrm{ml}$.

\section{Results}

Results of the experiment are summarized in terms of the amount of $\mathrm{NaCl}$ solution consumed by animals in the various groups in the training session (Day 15) and in the test session (Day 24). The amount consumed by the animals in the various groups in the training session have been pooled together because an ANOVA on amount consumed showed that differences between groups were not reliable $[F(6,31)=1.56]$. Pooling of the drinking scores was only for the purposes of the figure and not for any subsequent analyses.

Inspection of Figure 2 reveals that all groups, except Group SAL, showed large reductions in consumption of the $\mathrm{NaCl}$ solution between Days 15 and 24. This impression was supported by individual $t$ tests for correlated means on the amount consumed on the training and test days by animals in the various groups. Animals in Group LEM $[\mathrm{t}(4)=3.70$, $\mathrm{p}<.05]$, Group COF $[\mathrm{t}(6)=6.93, \mathrm{p}<.01]$, Group ALM $[\mathrm{t}(5)=9.27, \mathrm{p}<.01]$, Group SAC $[\mathrm{t}(4)=$ 4.26, $\mathrm{p}<.02$ ], Group VAR $[\mathrm{t}(4)=3.40, \mathrm{p}<.05]$, and Group WAT $[\mathrm{t}(4)=2.97, \mathrm{p}<.05]$ all showed reliable reductions in consumption. In contrast, animals in Group SAL [t(4) $=2.81, \mathrm{p}<.05]$ showed a reliable increase in consumption.

It is possible to view the amount consumed by animals in Group WAT as a baseline measure against which to assess the degree of extinction that

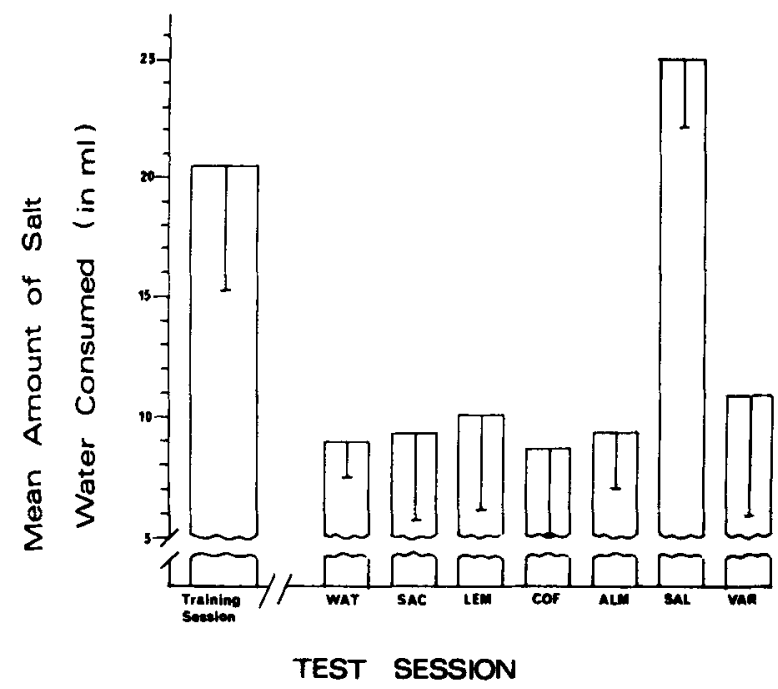

Figure 2. Mean amount of salt water consumed on the training day (pooled) and on a subsequent test session. Perpendicular lines are group standard deviations.

was caused by presentation of the various solutions. It is apparent from Figure 2 and the previous analysis that animals in Group WAT drank substantially less in the test session than they did in the training session, indicating that animals that were untreated with a distinctively flavored solution following conditioning had indeed formed an aversion to the $\mathrm{NaCl}$ solution. In order to make direct comparisons among the groups, the degree of aversion exhibited by animals in this and in the other groups was expressed in terms of a consumption ratio which was calculated by dividing the amount consumed by animals on the test day by the amount that they had consumed on the training day. A ratio of 1.0 indicates that animals drank the same amount in the two sessions, a ratio of less than 1.0 indicates that there was a reduction in consumption, while a ratio greater than 1.0 indicates that consumption increased. Group means and standard deviations are presented in Table 1 and show that animals in all groups, except in Group SAL, experienced reductions in consumption ranging from approximately $37 \%$ to $63 \%$, while animals in Group SAL exhibited a $35 \%$ increase in consumption. A one-way ANOVA on the ratio scores indicated that there

Table 1

Mean and Standard Deviation of the Ratio Scores (Test $\div$ Training)

\begin{tabular}{lrl}
\hline Group & Mean & SD \\
\hline WAT & .60 & .22 \\
SAC & .44 & .25 \\
LEM & .63 & .18 \\
COF & .37 & .19 \\
ALM & .47 & .10 \\
SAL & 1.35 & .26 \\
VAR & .57 & .27 \\
\hline
\end{tabular}


were reliable overall differences among the mean ratio scores $[\mathrm{F}(6,31)=14.50, \mathrm{p}<.01]$. Individual comparisons, using the Newman-Keuls procedure ${ }^{2}$ revealed that all of the ratios were reliably smaller than the one calculated for Group SAL (all ps $<.01$ ) but that none of the other means differed from each other.

This analysis indicates that all of the groups, except Group SAL, formed aversions that were not reliably different from those formed by animals in Group WAT. Thus there was no evidence of extinction in animals that drank solutions whose tastes differed from that of the conditioning solution. The fact that animals that were extinguished and trained with the same solution showed an increase in consumption (i.e., complete extinction of the aversion) indicates that the procedure employed did not preclude extinction of the aversion.

The results of the present experiment, then, show that extinction trials with solutions which differed from the test solution did not modify the association that had already formed between the $\mathrm{NaCl}$ solution and the aftereffects of the lithium chloride injection. These results are consistent with findings from the previous experiment, which showed that preexposure to the non- $\mathrm{NaCl}$ solutions had no effect on the subsequent associations between the $\mathrm{NaCl}$ solution and the $\mathrm{LiCl}$ injection. In addition, following from Pavlov's (1927, p. 56-57) experiments on secondary extinction, the present findings also suggest that animals did not perceive the taste of the $\mathrm{NaCl}$ solution as being similar in any way to the taste of the other non- $\mathrm{NaCl}$ solutions. Had the non$\mathrm{NaCl}$ solutions been perceived as being related to the $\mathrm{NaCl}$ solution, then there should have been evidence of extinction in Groups SAC, LEM, COF, ALM, and VAR.

\section{EXPERIMENT 3}

The results of the first two experiments indicate that exposure to solutions other than the test solution, either before or after conditioning, does not appear to have any effect on the association between the taste of the test solution and the aftereffects of the lithium chloride. Preexposure, however, to flavored solutions which differ from or are the same as the test solution does appear to interfere with the natural hesitancy of rats to ingest a novel solution.

One question that emerges from these latter findings concerns the time course in the reduction of neophobia under the various conditions of solution exposure. From Siegel's point of view, it is reasonable to expect that animals exposed to a single solution will, on successive presentations of that solution, drink increasingly more and that, up to a point, these animals will reduce their neophobic tendencies more rapidly than animals that are exposed to a changing series of solutions. This expectation is based on the presumption that it is easier for animals to characterize a single solution as being safe than to characterize each of a number of different solutions as being safe. From Capretta et al.'s point of view, however, it is possible that animals experiencing diversity in the tastes of the solutions would be more likely to ingest yet another novel flavored solution than animals that have not had the experience with diversity since, according to this position, one way to reduce neophobia is by providing animals with dietary diversity.

In the present experiment, we examined these possibilities by monitoring the amount consumed by animals that drank (1) familiar tap water, (2) a novel coffee solution, or (3) a series of four different flavored solutions on 8 successive days. Our interest was to discover at what point in time the amount consumed under the last two conditions became indistinguishable from each other and from the tap water condition. According to Siegel's position, consumption by animals that drink only coffee should approximate water consumption more rapidly than consumption by animals that receive a changing series of solutions. On the other hand, the position held by Capretta et al. would predict that the rate at which consumption by animals that receive a variable series of solutions approximates the amount of familiar tap water consumed should not differ from and even might surpass the rate at which neophobia is reduced in animals that receive only a single novel solution.

\section{Method}

Subjects. The subjects were 36 experimentally naive, male hooded rats from the same strain and supplier as in the previous experiments. Their weights ranged from 250 to $300 \mathrm{~g}$ at the start of the experiment.

Apparatus and Procedure. The cages, solutions, and calibrated drinking bottles used in this experiment were the same as those used in the previous experiments.

All animals were adaipted to a 10-min-per-day drinking schedule for at least 7 days. Food was continuously available throughout the experiment. Following the adaptation period, the animals were randomly assigned to one of three groups of 12 animals each. For the next 8 days, the animals in Group Water received tap water for $10 \mathrm{~min}$ per day, those in Group Coffee received $.75 \%(w / v)$ coffee solution for $10 \mathrm{~min}$, and those in Group Variable received one of four solutions for $10 \mathrm{~min}$. Presentation of solutions to the animals in Group Variable was arranged in such a way that the same solution was presented on 2 successive days. This was done in order to make it possible to assess the degree to which neophobia was reduced by comparing consumption on the first presentation with consumption on the second presentation. Furthermore, within Group Variable there were four subgroups ( $N=3$ per group) which were determined by the specific sequence of flavored solutions. On any given day, all animals within a subgroup received the same solution but animals in different subgroups received different solutions. This permitted the use of four different 
sequences and allowed us to partly counterbalance the presentation sequence so as to insure that the outcome was not peculiar to a specific sequence of solutions. For example, animals in Subgroup SALC received saccharin water (S) on the first 2 days of the experiment, almond water (A) on Days 3 and 4, lemon water (L) on Days 5 and 6, and coffee water (C) on Days 7 and 8. The order of presentation for Subgroup ACSL was almond, coffee, saccharin, and lemon; for Subgroup CLAS was coffee, lemon, almond, and saccharin.

Each of the animals in Groups Variable and Coffee were yoked to an animal in Group Water. By providing animals in Groups Variable and Coffee with an amount of tap water which equaled the difference between the amount of the flavored solution that they consumed and the total amount of tap water that their yoked animals in Group Water had consunned, it was possible to insure that total fluid intake by animals in Groups Variable and Coffee did not become depressed by a hesitancy to drink the distinctively flavored test solutions. The additional tap water was given to animals in Groups Variable and Coffee 2-3 $\mathrm{h}$ after the 10-min drinking period. With the exception of two cases, the water was typically consumed within $5 \mathrm{~min}$.

\section{Results}

The results of the present experiment are summarized in Figure 3 in terms of the mean amount of solution consumed by animals in the three groups on each of the 8 test days. A 3 (treatment) by 8 (test day) repeated-measures design ANOVA was calculated on the amount that each animal consumed on each of the test days. Results of this analysis produced a significant main effect due to Treatments $[F(7,33)=10.92, p<.01]$, a significant main effect due to Test Day $[F(7,231)=221.64, p<.01]$, and a significant interaction between these two factors $[F(14,231)=143.58, p<.01]$. A trend analysis

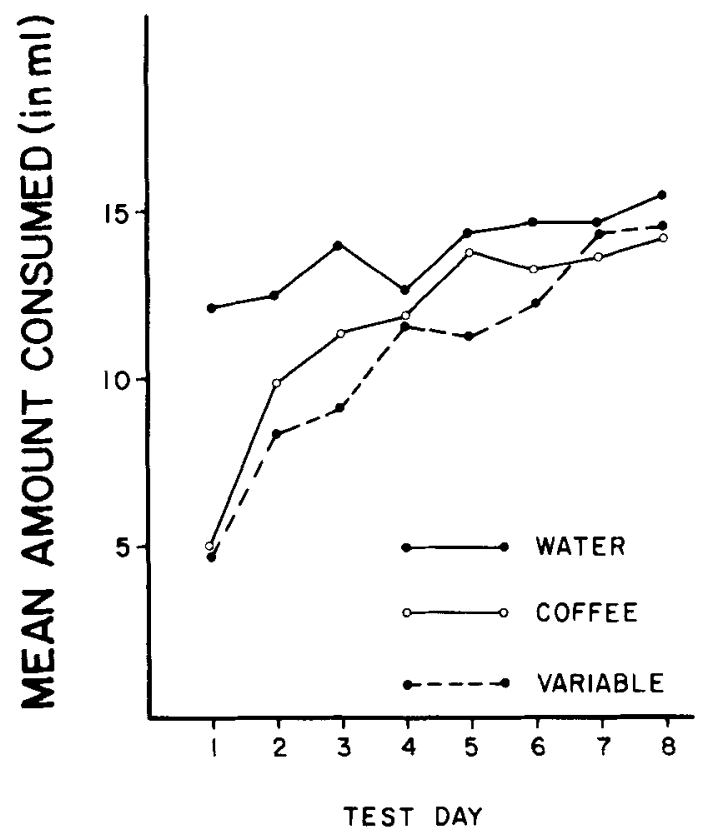

Figure 3. Amount consumed on successive presentations of a variable series of saccharin-, lemon-, almond-, and coffee-flavored water (Variable), of unflavored tap water (Water), or of coffeeflavored water (Coffee). using orthogonal polynomials on the curves presented in Figure 3 indicated that the curve for the Water group is best described by a linear function $[F(1,33)=16.12, p<.01]$. This, along with the outcome of a t test on correlated means which showed that consumption of water on Test Day 8 did not differ from that on Test Day 1, indicates that water consumption was stable for animals in Group Water.

In contrast, the functions that best describe the Coffee and Variable groups were quadratic $[F(1,33)$ $=8.14, \mathrm{p}<.01$, and $F(1,33)=4.66, \mathrm{p}<.05$, respectively. Additional comparisons of the amount consumed by animals in these two groups on each of the test days, using independent $t$ tests in which the mean square error for between groups was used to estimate the standard error of the difference between means, showed that none of the day-today differences among the groups was significant. Thus the pattern of consumption by animals in Group Variable was indistinguishable from that of animals in Group Coffee. Moreover, post hoc comparisons involving individual $F$ tests (Weiner, 1962) showed that animals in Group Water drank reliably more than the average of the other two groups on only the first $[F(1,33)=8.61, p<.01]$ and second $[F(1,33)=5.22, p<.05]$ test days. After that, consumption by animals in both the Variable group and the Coffee group was indistinguishable from that of animals in the Water group.

The results of the present experiment show that neophobia was reduced rather quickly and that the rate of reduction was the same for animals exposed to the same novel solution as it was for those exposed to a changing series of solutions. According to the results of the present experiment, from the third presentation onward, animals that received a novel coffee solution and those that drank a changing series of solutions consumed as much liquid as animals that drank familiar tap water. This finding would appear to support more closely Capretta's conclusion that dietary diversity per se is an important determinant of whether or not animals consume novel substances, since, in the present instance, animals in Group Variable drank as much of a completely novel solution on each pair of test days following the first two as animals in the remaining groups drank of a relatively novel coffee solution or of a completely familiar tap water solution.

\section{GENERAL DISCUSSION}

The results of the preceding experiments show that exposure to solutions other than the test solution can reduce neophobia to the test solution, but it does not appear to interfere with associations involving the taste of the test solution and the aversive 
aftereffects of a lithium chloride injection. In contrast, exposure to the test solution not only reduces neophobia but it also appears to interfere with tastelithium chloride associations.

The fact that exposure to solutions other than the test solution is selectively effective in interfering with neophobia but not with conditioning suggests that Siegel's (1974) explanation of preexposure effects on neophobia may be too restrictive. According to Siegel, animals that learn, through preexposure, that a solution or one similar to it is safe should not be neophobic to the test solution and they should form weaker aversions to it. The reason that they should form weaker aversions to it is that, having learned during preexposure that the solution is a safe one, it is now more difficult for them to learn that it will lead to noxious postingestional consequences. The fact that we were able to reduce neophobia by preexposing animals to solutions other than the test solution without disrupting conditioning shows that the two phenomena need not be coupled and, as a result, suggests that learned safety may not have transferred between the discriminable solutions used in the present experiment.

A limiting factor in the preceding conclusion, however, is that the one-bottle test may not have been as sensitive a measure of conditioning as it was of neophobia. As a result, preexposure to non $-\mathrm{NaCl}$ solutions may appear to have been effective in reducing neophobia but not conditioning only because the one-bottle test was more sensitive to effects of the experimental manipulations on neophobia than it was to the effects of the same manipulations on conditioning. Although the present experiments do not allow for a direct assessment of this possibility, it is important to note that the one-bottle test was sensitive enough to measure the differential effects of preexposure to tap water and/or the $\mathrm{NaCl}$ solution on conditioning and extinction. Preexposure to tap water did not affect neophobia or conditioning while preexposure to the $\mathrm{NaCl}$ solution interfered with both. Similarly, extinction with tap water did not disrupt the already established taste-lithium chloride association while extinction with the $\mathrm{NaCl}$ solution totally disrupted that association. The reason is not readily apparent why the one-bottle test should be sensitive enough to detect differences involving these two solutions but not differences involving the other flavored solutions employed.

In addition to the reservations raised by $\mathrm{Ex}$ periments 1 and 2 about Siegel's interpretation of how neophobia is reduced, elements of Experiment 3 appear not to be totally congruent with Capretta et al.'s position that neophobia is reduced (i.e., novel solutions are more readily accepted) because animals are prepared to accept novel solutions by previous dietary diversity. It was observed on the 3rd test day of Experiment 3 that animals in Groups Coffee and Variable consumed as much of their respective novel solutions as animals in Group Water consumed of tap water. One interpretation of this finding is that neophobia was completely reduced at that point in time. In this regard, it is important to point out that for animals in Group Variable the solution presented on the 3rd test day was completely novel while animals in Group Coffee had had two prior presentations of the coffee solution and animals in Group Water were drinking highly familiar tap water. Thus, after only two presentations of a single solution, consumption of another novel solution by animals in Group Variable did not differ reliably from consumption of a relatively familiar coffee solution or from consumption of highly familiar tap water. Equally as interesting is the fact that the same pattern was upheld on Test Days 5 and 7, corresponding to the days when animals in Group Variable received other novel solutions. The point here is simply that the Test Day 3 findings, in particular, appear to show that extensive dietary diversity is not a prerequisite for increasing the acceptance of novel solutions. Our results, along with those reported by Siegel (1974), suggest that neophobia is reduced rather quickly in adult organisms and that extensive experience may not be required to produce the transfer effects noted by Capretta et al.

It is possible to interpret the present pattern of results, including those of Experiment 3, within the framework of an explanation which holds that novel or unfamiliar tastes are avoided because novelty itself is aversive (e.g., Domjan, 1976). Within this framework, it is unimportant whether or not animals experience the flavor of the test solution prior to tests for neophobia. As long as a novel test solution is not perceived as being unusual animals will ingest it readily. Apparently, one way to reduce the aversiveness of an unfamiliar test solution is by preexposing animals to other novel solutions. In terms of the findings reported in the present experiments as well as those reported elsewhere, the aversiveness of novelty can be reduced rather quickly following the presentation of a single solution. At the same time, however, our findings show that having reduced the aversiveness of one novel solution by exposing animals to another novel solution does not appear to interact with associations between ingestion of the now nonaversive novel solution and the noxious effects of a lithium chloride injection.

Finally, it should be noted that the present position does not in any way conflict with the observation that preexposure to the test solution results both in a reduction of neophobia and in a weakening of conditioned aversions. According to 
the novelty position, reductions in neophobia are the result of a general process which, as has been noted above, is produced by reductions in the aversiveness of the novel test solution. The interference with conditioning, on the other hand, depends on another, more specific process which may be similar to latent inhibition (e.g., Best, 1975; Revusky, 1971). Thus, when animals have had experience with a solution whose flavor is the same as that of the test solution prior to conditioning, it is possible to interfere with the natural hesitancy to consume the test solution as well as with the association between the taste of the test solution and the effects of the aversion-inducing treatment. The former occurs because of a reduction in the novelty, while the latter occurs because the salience of the taste cues has been reduced.

\section{REFERENCE NOTE}

1. Braveman, N. S. Stimulus specificity in reduced neophobia and the conditioning of taste aversions. Paper presented at the 17th Annual Meeting of the Psychonomic Society, St. Louis, 1976.

\section{REFERENCES}

Barnetr, S. A. The rat: A study in behavior. Chicago: Aldine, 1963.

BEST, M. R. Conditioned and latent inhibition in taste-aversion learning: Clarifying the role of learned safety. Journal of Experimental Psychology: Animal Behavior Processes, 1975, 1, 97-113.
Capretta, P. J., Petersik, T. T., \& Stewart, D. J. Acceptance of novel flavor is increased after early experiences with diverse tastes. Nature, 1975, 254, 689-694.

Domun, M. Determinants of the enhancement of flavoredwater intake by prior exposure. Joumal of Experimental Psychology: Animal Behavior Processes, 1976, 2, 17-27.

Pavlov, I. P. Conditioned reflexes. New York: Dover, 1927.

Revusky, S. H. The role of interference over a delay. In W. K. Honig \& H. James (Eds.), Animal memory. New York: Academic Press, 1971.

SIEGEL, S. Flavor pre-exposure and "learned safety." Journal of Comparative and Physiological Psychology, 1974, 87, 1078-1082.

WEINER, B. J. Statistical principles in experimental design. New York: McGraw-Hill, 1962.

\section{NOTES}

1. It is possible to argue that the most adequate test of the present hypothesis necessitates the use of a within-subjects design. However, Braveman (Note 1) has reported nearly identical findings when such a design was employed. This would appear to indicate that the phenomena of reduced neophobia without reduced conditioning following familiarization to solutions whose tastes differed from that of the test solution is a general one and not a function of the experimental design employed.

2 . Since the number of subjects per group (n) was not the same, it was not possible to use the formula $\sqrt{\text { nMS error to calculate }}$ the critical values for the Newman-Keuls (Weiner, 1962). Instead, $\mathrm{n}$ was estimated by using a harmonic value in which $\tilde{\mathrm{n}}=$ $\mathrm{G} /\left(1 / \mathrm{n}_{1}+1 / \mathrm{n}_{2}+\ldots+1 / \mathrm{n}_{\mathrm{g}}\right)$, where $\tilde{\mathrm{n}}=$ the harmonic number of subjects per group, $\mathrm{G}=$ number of groups, and $n_{q}=$ number of subjects in the Gth group.

(Received for publication September 22, 1977; revision accepted April 3, 1978.) 